\title{
Uma estratégia online para degradação de serviço com QoS proporcional em Redes Ópticas Elásticas
}

\author{
Alex S. Santos ${ }^{1}$, Juliana de Santi $^{2}$, Gustavo B. Figueiredo ${ }^{1}$ \\ ${ }^{1}$ Instituto de Matemática - Universidade Federal da Bahia (UFBA) \\ Av. Adhemar de Barros, s/n, Ondina - 40170-115 - Salvador - BA - Brasil \\ ${ }^{2}$ Departamento de Informática - Universidade Tecnológica \\ Federal do Paraná (UTFPR)
}

santos.alex@ufba.br, jsanti@utfpr.edu.br, gustavo@dcc.ufba.br

\begin{abstract}
Elastic Optical Networks represent a new approach to dealing with high data traffic in high performance networks by offering bandwidth closer to that requested by users. In the current literature it is possible to perceive the absence of online strategies for degradation of service, which consists of an approach used to deal with the lack of available optical resources. This paper proposes an online strategy for service degradation using the proportional QoS model. This strategy aims to reduce the probability of blocking requests caused by lack of optical resources and to give a better treatment for different classes of service. In addition, pairs of source-destination nodes were modeled as a queuing system operating over the Generalized Processor Sharing (GPS) policy with Leaky Bucket control admission as the goal of quantifying the impact of degradation on lightpaths. The results show that the proposed strategy can reduce the probability of blocking by giving network operators greater control among different classes of degraded services.
\end{abstract}

Resumo. As Redes Ópticas Elásticas representam uma nova abordagem para lidar com o elevado tráfego de dados nas redes de alto desempenho, ao oferecer largura de banda mais próxima da solicitada pelos usuários. Na literatura atual é possivel perceber a ausência de estratégias online para degradação de serviço, que consiste em uma abordagem utilizada para lidar com a falta de recursos ópticos disponíveis. Neste trabalho é proposto uma estratégia online para degradação de serviço usando o modelo de QoS proporcional. Esta estratégia visa reduzir a probabilidade de bloqueio causada por falta de recursos ópticos, e dar um melhor tratamento para diferentes classes de serviço. Além disso, os pares de nós origem-destino foram modelados como um sistema de enfileiramento operando sobre a política Generalized Processor Sharing (GPS) com admissão de controle Leaky Bucket com o objetivo de quantificar o impacto da degradação nos lightpaths. Os resultados obtidos mostram que a estratégia proposta pode reduzir a probabilidade de bloqueio fornecendo aos operadores de rede maior controle entre diferentes classes de serviços degradadas.

\section{Introdução}

A quantidade de tráfego de dados no núcleo da rede tem duplicado a cada dois anos e estudos mostram que o crescimento continuará a exibir esta característica exponencial 
[Jinno et al. 2009]. Como consequência, as operadoras de rede terão que transportar uma quantidade variada de tráfego de dados que pode ir de dezenas de Gigabits até Terabits por segundo de forma rentável e escalável [Jinno et al. 2009, Zhang et al. 2013]. Para suportar esta demanda crescente é preciso que as redes de transporte sejam capazes de lidar com diferentes volumes de tráfego de forma eficiente. Redes WDM (do inglês Wavelengthdivision Multiplexing (WDM)) enfrentam dificuldades para atender tais demandas devido à baixa granularidade dos recursos espectrais oferecidos. Assim, uma nova abordagem denominada Rede Óptica Elástica (do inglês Elastic Optical Network (EON)) surge como uma tecnologia promissora para lidar com as novas demandas de serviços e aplicações. Para utilizar o espectro óptico de maneira eficiente, as redes EONs realizam alocação de canal de forma flexível, na forma de slots de frequência com granularidade de 12,5G $\mathrm{Hz}$. Assim, as EONs são capazes de prover segmentação e agregação de recursos espectrais, acomodação eficiente das múltiplas taxas de dados e variação elástica dos recursos ópticos alocados [Jinno et al. 2009, Tomkos et al. 2014, Napoli et al. 2015].

Apesar da sua capacidade de transmissão e adaptação, algumas situações como desastres naturais ou ataques à infraestrutura podem provocar falhas e ocasionar perda brusca de recursos, gerando, consequentemente, congestionamentos. Nesse caso, uma das propostas para lidar com a falta de recursos ópticos é o provisionamento com degradação de serviço, onde um nível degradado de serviço é oferecido aos clientes visando reduzir o número de requisições bloqueadas quando a rede se torna congestionada. Dessa forma, determinadas conexões podem ser degradadas, isto é, ter sua largura de banda reduzida para aumentar o número de requisições aceitas [Zhong et al. 2016].

Ao realizar a degradação, é preciso garantir que a redução da largura de banda não afetará a Qualidade de Serviço (do inglês Quality of Service (QoS)) oferecida ao cliente. Além disto, é preciso assegurar que determinadas classes de serviço não sejam mais penalizadas que outras. A maioria das propostas existentes na literatura para a degradação de serviço, não diferenciam as necessidades que cada classe de serviço pode exigir. Além disso, utilizam Programação Linear Inteira (do inglês Integer Linear Programming (ILP)) baseado em um cenário estático onde todas as requisições são conhecidas antecipadamente.

Este artigo apresenta uma estratégia online para degradação de serviço com QoS proporcional em Redes Ópticas Elástica que visa minimizar a probabilidade de bloqueio de novas requisições de conexão. Nessa abordagem, as requisições são divididas em classes de serviço e o QoS proporcional é utilizado para manter a proporção entre o número de requisições degradadas de cada classe de serviço. Esta medida é adotada para oferecer maior controle sobre as classes de serviço aos operadores de rede. Além disso, para garantir que a degradação não provocará a violação do tempo de transmissão especificado para cada requisição, os lightpaths presentes na rede EON foram modelados como um Generalized Processor Sharing (GPS) com controle de admissão Leaky Bucket. Os resultados obtidos mostram que o algoritmo proposto tem a capacidade de reduzir a probabilidade de bloqueio e dar mais flexibilidade no tratamento de diferentes classes de serviço.

O restante do artigo está organizado da seguinte forma: A Seção 2 apresenta a tecnologia das Redes Ópticas Elásticas. A Seção 2.1 descreve a degradação de serviço em EON. A Seção 3 apresenta o modelo de QoS proporcional. Na Seção 4 é apresentado o algoritmo proposto. Na Seção 5 são apresentados os resultados obtidos. Por fim, na 
Seção 6 o artigo é concluído.

\section{Redes Ópticas Elásticas}

As Redes Ópticas Elástica surgiram com o objetivo de melhorar a utilização dos recursos ópticos disponíveis oferecendo larguras de banda mais próximas das requisitadas pelos clientes. Ao contrário de redes WDM que fornecem largura de banda fixa, muitas vezes subutilizando os recursos ópticos, as Redes Ópticas Elástica têm a capacidade de ajustar sua largura de banda e formato de modulação para valores mais adequados ao solicitado pelo cliente. Para isso, o espectro é dividido em slots de frequência e a alocação de conexões é realizada utilizando diferentes quantidades de slots [Chatterjee et al. 2015].

Para aumentar a eficiência na utilização dos recursos espectrais disponíveis, as Redes Ópticas Elásticas utilizam slots de frequência com tamanho reduzido. Os slots de frequência possuem largura de $12,5 \mathrm{GHz}$ e podem ser combinados para acomodar os lightpaths que são as conexões ópticas entre pares de nós origem-destino. As EONs utilizam a tecnologia de modulação OFDM (do inglês Orthogonal FrequencyDivision Multiplexing), que é considerada uma forte candidata a ser empregada na transmissão óptica de alta velocidade. Esta tecnologia é adotada para a transmissão multiportadora onde os dados transmitidos em alta velocidade são divididos em múltiplos canais paralelos de mais baixa velocidade. Além disso, ela permite que espectros de subportadoras individuais se sobreponham, graças à ortogonalidade presente entre eles [Gerstel et al. 2012, Zhang et al. 2013]. A OFDM é adotada em diversas tecnologias de rede com e sem fio tais como 802.11a/g Wi-Fi, 802.16 WiMAX, LTE, DSL e outras.

Para a criação de um lightpath diferentes formatos de modulação podem ser adotados. Uma característica chave para escolher qual modulação adotar está relacionada ao alcance transparente que determina uma restrição modulação-distância, pois modulações com maior eficiência espectral tendem a ter menor alcance. $\mathrm{O}$ alcance máximo dos principais formatos de modulação com qualidade aceitável e sem a necessidade de regeneração de sinal podem ser observados na Tabela 1.

Tabela 1. Formatos de modulação. Adaptado de [Anoh et al. 2017].

\begin{tabular}{|l|c|c|} 
Modulação & Alcance transparente $(\mathrm{Km})$ & Eficiência espectral (b/s/Hz) \\
\hline BPSK & 4000 & 1 \\
\hline QPSK & 2000 & 2 \\
\hline 8-QAM & 1000 & 3 \\
\hline 16-QAM & 500 & 4 \\
\hline
\end{tabular}

Segundo [Tomkos et al. 2014], a base que possibilita o funcionamento das Redes Ópticas Elásticas é composta por: flexible transceivers e flexible optical switches. Os flexible transceivers têm a capacidade de compartilhar seus recursos entre múltiplos nós fonte e destino através da separação em vários "transceivers virtuais", onde cada um atende a uma demanda específica. Os Flexible optical switches realizam a comutação de supercanais ópticos com largura de banda variável em uma fina granularidade. Outra característica importante é a sua capacidade de realizar Optical grooming, que é a agregação e distribuição do tráfego diretamente na camada óptica. Na Figura 1 é possível observar a disposição destes componentes na arquitetura óptica elástica. 


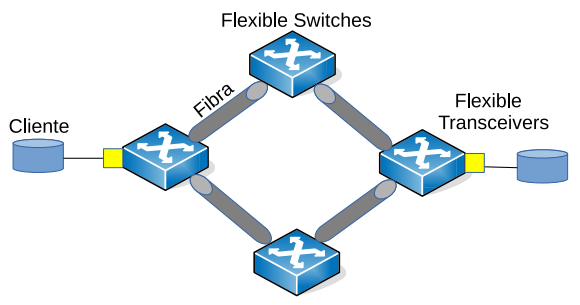

Figura 1. Representação da Arquitetura EON. Adaptado de [Jinno et al. 2009].

\subsection{Degradação de Serviço em redes ópticas elásticas}

Um dos principais objetivos de provedores de rede é manter sua infraestrutura lucrativa e a fidelidade de seus clientes. Com o surgimento de diversas aplicações que demandam a transmissão de elevado volume de dados, novas estratégias precisam ser definidas para garantir a qualidade de serviço, pois cada conjunto de aplicações possui requisitos específicos, tais como prioridade, segurança e tempo de transmissão, que devem ser atendidos para proporcionar qualidade de serviço. Aumentar indefinidamente a largura de banda não é uma medida adequada a ser tomada, pois ela apresenta um alto custo e não contempla as diferentes características que cada serviço pode apresentar. Uma das abordagens para solucionar este problema é oferecer provisionamento com degradação de serviço. Nesta forma de provisionamento, quando a rede se torna congestionada, determinados clientes podem receber largura de banda menor do que a solicitada. Tal medida visa evitar que novas requisições sejam bloqueadas devido à incapacidade da rede em alocar recursos [Zhong et al. 2016].

Segundo [Savas et al. 2014a], a degradação de serviço está relacionada com a redução de recursos para um determinado serviço versus a quantidade de recursos utilizados em sua operação normal. Para realizar a degradação de serviço, é importante que questões como qual requisição deve ser degradada ou o quanto uma determinada requisição pode ser degradada sejam respondidas para garantir a qualidade de serviço fornecida ao usuário. A realização da degradação na camada óptica está relacionada à redução da quantidade de espectro ocupada por um lightpath em conformidade com o aumento da modulação de maneira a garantir a sua taxa de dados. A degradação óptica deve obedecer à restrição da modulação-distância, pois modulações de alto nível tendem a ter menor alcance.

A degradação pode ser realizada de duas formas: Degradação Single-side e Degradação Double-side. Estas duas abordagens podem ser visualizadas na Figura 2. Na Figura 2(a) a Conexão 1 ocupa 4 slots e a Conexão 2 ocupa 3 slots, não restando mais slots para o estabelecimento da nova Conexão 3. Na Figura 2(b) é realizada a Degradação Single-side, onde a Conexão 2 perde 1 slot de frequência para que a Conexão 3 seja estabelecida. Na Figura 2(c) é realizada a Degradação Double-side, onde a Conexão 1 e a Conexão 2 perdem 1 slot para o estabelecimento da Conexão 3.

\subsection{Trabalhos Relacionados}

Devido a grande importância das redes ópticas de alto desempenho, diversas pesquisas têm surgido com o objetivo de maximizar a sua utilização. A seguir são apresentados alguns trabalhos relacionados à degradação de serviço e a forma como os autores abordam o problema. 


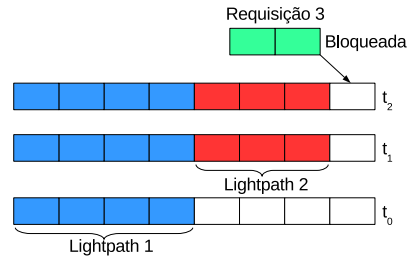

(a) Falta de slots

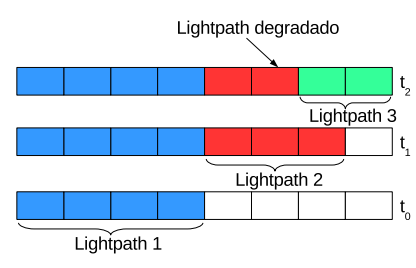

(b) Degradação Single-side

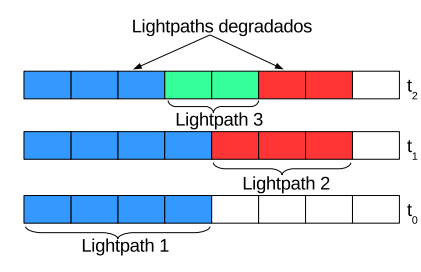

(c) Degradação Double-side

Figura 2. Exemplos de degradação de serviço.

Em [Vadrevu et al. 2014] é estudado o provisionamento de serviços degradados em redes Mixed-Line-Rate WDM Backbone, as quais suportam caminhos ópticos com diferentes taxas que podem ser de $10 \mathrm{Gbs}, 40 \mathrm{Gbs}, 100 \mathrm{Gbs}$, etc. Os autores estudam o conceito de proteção parcial com roteamento multi-caminhos, onde uma fração da banda requisitada é provida para o usuário mediante cenário de falha, e a banda total requisitada é provida nos períodos de operação normal. Entretanto, em situações de escassez de recurso óptico, esta abordagem pode não alcançar o objetivo desejado de reduzir a taxa de bloqueio de novas requisições.

Os autores em [Savas et al. 2014b] apresentam uma estratégia para admissão de conexões com serviço degradado quando não for possível admiti-las com a largura de banda total requisitada. Se a degradação da nova requisição não for suficiente para que ela seja atendida, conexões estabelecidas podem ser degradadas para que a nova conexão seja provisionada. Além disso, é proposto um processo de atualização que restaura, quando possível, a largura de banda total da requisição com o objetivo de melhorar a qualidade de serviço de acordo com as condições atuais da rede. Neste trabalho, verifica-se que a degradação da QoS prejudica a experiência de uso da rede.

[Zhong et al. 2016] utiliza a abordagem de QoS-assegurado para investigar o provisionamento com degradação de serviço em redes multicamadas. Os autores dividem o problema em duas etapas: roteamento com degradação e alocação com degradação de recursos.

Os trabalhos apresentados mostram diferentes abordagens para provisionamento de requisições de conexão com degradação de serviço em diferentes tipos de redes ópticas. Nossa abordagem se diferencia das demais por empregar a utilização do modelo de QoS proporcional para prover justiça entre as classes degradadas.

\section{O Modelo de QoS Proporcional}

Os serviços oferecidos na Internet pelos diversos provedores de conteúdo possuem diferentes requisitos para funcionarem de maneira adequada. Neste cenário, novas propostas surgiram para prover garantias de Qualidade de Serviço. Uma das abordagens utilizadas para solucionar este problema é a Differentiated services-DiffServ. Nesta abordagem, os pacotes são classificados em um pequeno número de classes de serviço de acordo com os seus requisitos. Para alcançar este objetivo, o DiffServ não foca em um pacote individual de um determinado fluxo, mas em tráfegos agregados, isto é, um vasto conjunto de fluxos com requisitos de serviços similares. Entretanto, o modelo DiffServ garante somente que o tráfego da classe de alta prioridade receberá um melhor serviço que a classe de baixa prioridade. O modelo de QoS proporcional é uma solução con- 
trolável, consistente e escalável que ganhou atenção como uma solução efetiva para a diferenciação quantitativa de serviços em redes IP. Sua maior característica é a capacidade de oferecer aos operadores de rede um controle ajustável e consistente de classes de serviço [Dovrolis and Ramanathan 1999, Chen et al. 2003].

Neste modelo, as medidas de desempenho para o encaminhamento de pacotes locais em cada salto são proporcionais a parâmetros específicos de diferenciação de classe definidas pelo operador de rede. Segundo [Chen et al. 2003], considerando $q_{i}$ uma métrica QoS de interesse e $s_{i}$ o fator de diferenciação para a classe $i$, o modelo de QoS proporcional fornece:

$$
\frac{q_{i}}{q_{j}}=\frac{s_{i}}{s_{j}},(i, j=1 \ldots N)
$$

Para um sistema real, não há garantias de que os fatores proporcionais possam ser iguais aos valores especificados pela razão de diferenciação. Além disso, para um curto período de tempo $\tau$ tem-se:

$$
\frac{\bar{q}_{i}(t, t+\tau)}{\bar{q}_{j}(t, t+\tau)}=\frac{s_{i}}{s_{j}},(i, j=1 \ldots N),
$$

onde $\bar{q}_{i}(t, t+\tau)$ é uma métrica QoS de interesse.

A diferenciação entre certas classes de serviço pode ser ajustada com um desvio limitado. Além disso, se $\tau$ é pequeno o suficiente, o tráfego em uma classe que possui alta prioridade receberá melhor serviço que uma classe de baixa prioridade mesmo se houver flutuação de carga. O modelo de QoS proporcional pode ser controlado pelos operadores de rede através de parâmetros de qualidade de diferenciação (do inglês Quality Differentiation Parameters (QDPs)) [Dovrolis and Ramanathan 1999, Chen et al. 2003].

\section{Abordagem proposta}

Nesta seção é descrita a abordagem online utilizada para o provisionamento de requisições com degradação de serviço em Redes Ópticas Elástica. Em nosso cenário, as requisições são definidas como tuplas $r_{i}=\left(s, d, c, b, w, D_{M}\right)$, onde $s$ e $d$ são os pares de nós origemdestino, $c$ é a classe de serviço da requisição, $b$ é a quantidade de dados a ser transmitida pela respectiva requisição, $w$ é a largura de banda requisitada e $D_{M}$ é o tempo máximo permitido para a requisição realizar a transmissão dos dados. A requisição de conexão é atendida pelo lightpath estabelecido sobre a menor rota entre os nós origemdestino. Além disso, atribui-se a modulação adequada para o estabelecimento do lightpath de forma transparente, respeitando a restrição modulação-distância. Desta forma, como o uso de modulações com maior alcance aumentará a quantidade utilizada de slots de frequência por uma requisição, e modulações com maior eficiência espectral podem não atender de forma transparente a distância do caminho origem-destino, a degradação de serviço é realizada reduzindo o número de slots do lightpath escolhido sem mudança da modulação adotada previamente.

Na próxima seção será descrito como a abordagem sugerida pode ajudar as operadoras de rede a decidir, dentre os lightpaths estabelecidos ao longo de uma determinada rota, qual caminho óptico deve ser degradado e o quanto deve ser degradado. 


\subsection{Algoritmo Proposto}

Nesta seção, apresenta-se o algoritmo proposto, denominado Degradação Mínima com QoS proporcional (MDP-QoS), para realizar degradação de serviço com QoS proporcional. O principal objeto deste algoritmo é degradar requisições que chegam à rede, ou aquelas já estabelecidas para reduzir a probabilidade de bloqueio de novas requisições devido à indisponibilidade de recursos. A degradação de serviço segue o modelo de QoS proporcional para fornecer maior controle entre as classes de serviço degradadas, isto é, evitar que uma classe de serviço seja mais penalizada que outra. Além disto, a extensão do tempo de transmissão causada pela degradação não deve ultrapassar o tempo máximo permitido para cada requisição.

O MDP-QoS é formalmente descrito em Algoritmo 1. Quando uma requisição $r_{i}$ chega, é escolhido o menor caminho entre os nós origem-destino utilizando o algoritmo de Dijkstra (Linha 2). Posteriormente, na Linha 3, define-se qual modulação deve ser utilizada, de acordo com a distância do caminho escolhido, e calcula-se a quantidade de slots de frequência necessários de acordo com a Equação 3:

$$
N_{s}=\left\lceil\frac{w}{M \cdot C}\right\rceil+N_{G B}
$$

onde $w$ representa a largura de banda solicitada, $M$ representa o formato de modulação, $C$ representa a largura de um slot de frequência e $N_{G} B$ é a quantidade de slots utilizados como banda de guarda.

Caso existam $N_{s}$ slots de frequência disponíveis no caminho $s$ - $d$, então a nova requisição $r_{i}$ é aceita (Linha 5). Em caso de falta de recursos, escolhe-se qual classe de serviço $c_{i}$ deve ser degradada de forma a manter a proporcionalidade entre classes de serviço (Linha 7). Se a nova requisição pertence a classe de serviço escolhida, então o algoritmo tenta degradar a requisição que acaba de chagar na rede (Linha 8-12). Neste processo, remove-se o número máximo de slots de frequência de $r_{i}$ sem provocar a violação do deadline estabelecido para a requisição. Caso a degradação da requisição $r_{i}$ não seja $o$ suficiente para sua acomodação, tenta-se degradar requisições já estabelecidas. Primeiro tenta-se realizar uma degradação Single-side, com o objetivo de afetar um menor número de requisições. Escolhe-se uma requisição $r_{j}$ já estabelecida que possua o mesmo par de nós $s$ - $d$ e, que ao ser degradada, possa ceder a quantidade de slots necessários para o provisionamento da requisição $r_{i}$ (Linha 17-21). Caso tal requisição não seja encontrada, então tenta-se realizar uma degradação Double-side, onde primeiro escolhe-se a requisição com o mesmo par de nós $s$ - $d$ que possa perder a maior quantidade de slots para definir onde a degradação Double-side será feita com o objetivo de maximizar as chances de sucesso.

Quando se realiza a degradação de requisições que chegam na rede ou a degradação Single-side, tenta-se manter a proporcionalidade entre classes de serviços, isto é, a requisição só é degradada se ela pertencer à classe escolhida nas Linhas 7 e 14. Por outro lado, quando é realizada a degradação Double-side, com o objetivo de aumentar as possibilidades de sucesso ao realizar esta ação, não se obedece mais a classe de serviço a qual cada requisição degradada pertence, sendo contabilizado para cada classe de serviço a degradação de uma requisição como nas Linhas 10 e 20. 


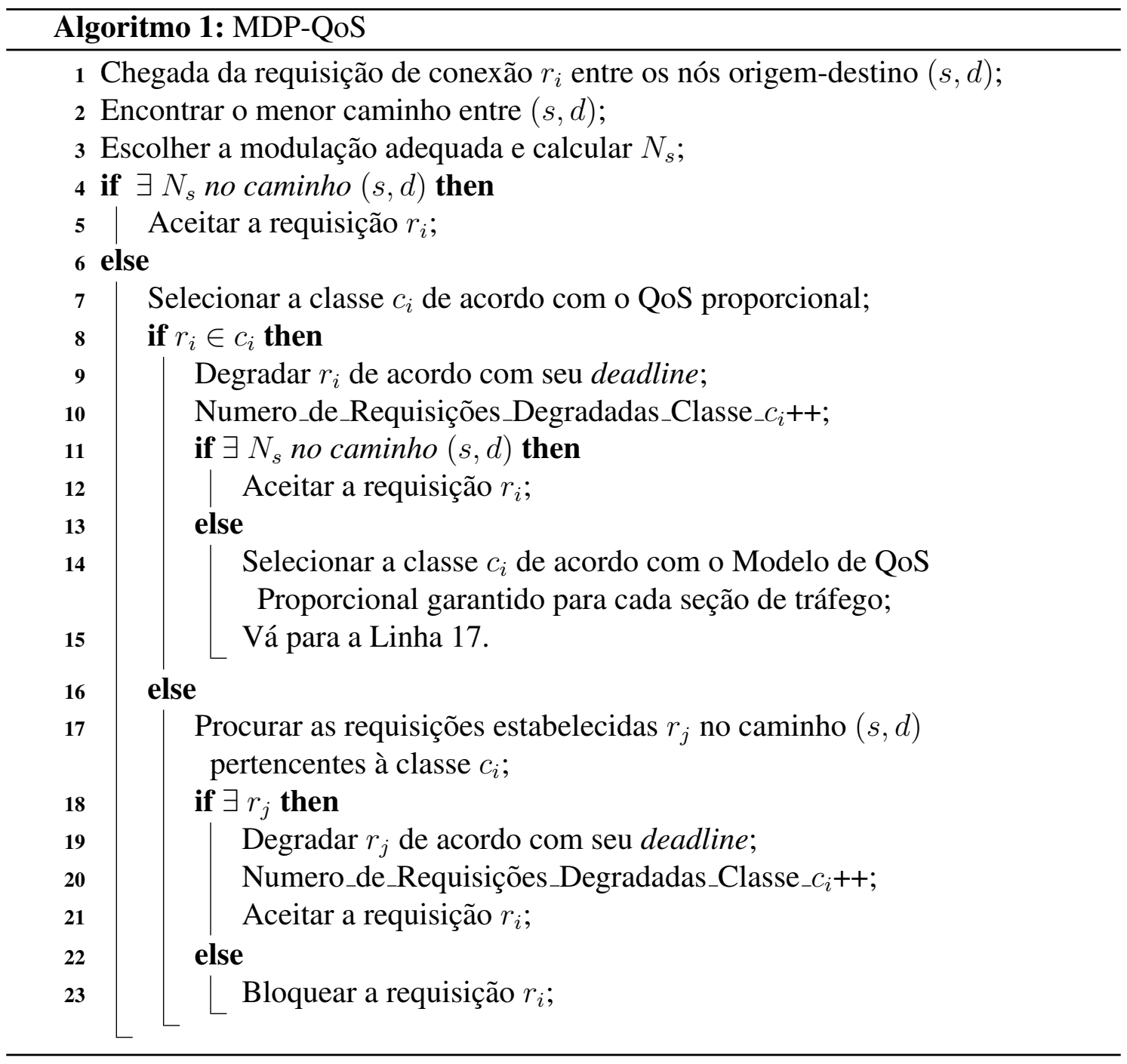

\subsection{Controle do atraso}

Como mencionado nas seções anteriores, a degradação de serviço, isto é, a atribuição de largura de banda menor que a solicitada, pode provocar a extensão do tempo de transmissão das requisições. Para garantir que o atraso máximo (delay) permitido para um lightpath é respeitado ao realizar a degradação, o conjunto de lightpaths provisionados foi modelado como um sistema de enfileiramento operando sobre a política GPS. Esta política consegue oferecer aos operadores de rede um conjunto sólido de equações que podem ser usadas para prover informações sobre diferentes lightpaths. Em um servidor GPS o fluxo dos clientes são servidos bit-a-bit de acordo com seus pesos normalizados. Ele oferece garantias de taxa de transferência e limites de atraso se os fluxos recebidos são atendidos seguindo o controle de admissão Leaky Bucket. Desta forma é possível estimar o impacto causado pelo provisionamneto de uma nova requisição sobre lightpaths já estabelecidos [Yap et al. 2012].

Em um servidor GPS que opera a uma taxa $R$, é garantido para cada seção de trafego $i$ um fluxo $R_{i}$ dado por: 


$$
R_{i}=\frac{\phi_{i}}{\sum_{y} \phi_{y}} R
$$

onde $\phi_{1}, \phi_{2}, \ldots, \phi_{3}$ são números reais positivos que representam os pesos associados a cada fluxo $i$. Em uma EON, o peso que corresponde a taxa de transmissão do lightpath $l$ é dado por:

$$
\phi_{l}=\frac{N_{l} T_{l}}{R},
$$

onde $N_{l}$ representa o número de slots de frequência do lightpath $l, T_{l}$ representa a taxa de transmissão de um slot de frequência, de acordo com o formato de modulação utilizado pelo lightpath $l$, e $R$ representa a largura de banda total utilizada entre os pares de nós origem-destino. Esta modelagem pode ser observada na Figura 3.

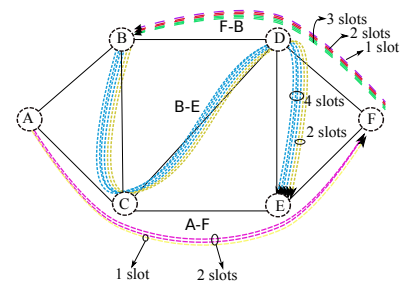

(a) Rede Óptica Elástica

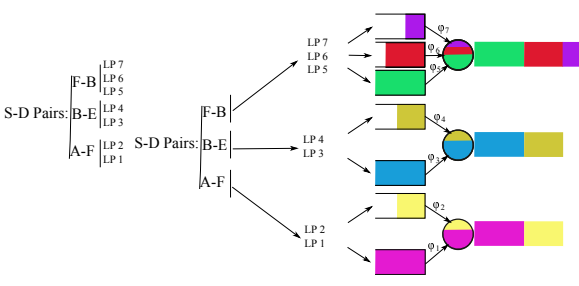

(b) Modelo GPS

Figura 3. Mapeamento da Rede Óptica Elástica como um modelo GPS.

Na Figura 3(a) é possível observar 3 pares de nós origem-destino (A-F, B-E, F-B). O primeiro usa a rota (A-C-E-F) e possui dois lightpaths provisionados (LP1 e LP2). Já o segundo usa a rota (B-C-D-E) e tem dois lightpaths provisionados (LP3 e LP4). Por fim, o terceiro usa a rota (F-D-B) e possui três lightpaths provisionados (LP5, LP6, LP7). A Figura 3(b) mostra estes lightpaths representados no modelo GPS proposto. Para cada lightpath é dado um peso relacionado ao seu número de slots de frequência de acordo com a Equação 6 [Yap et al. 2012].

$$
D \leq \frac{\sigma_{i}}{\rho_{i}}<\frac{\sigma_{i}}{R_{i}}
$$

Em um sistema GPS com Controle de Admissão Leaky Bucket (CALB), um fluxo $i$ é admitido se $\sum_{i} \rho_{i}<R$ e $\sum_{i} \sigma_{i}<B$, onde $\sigma_{i}$ é o limite superior para o processo de chegada, $\rho_{i}$ é taxa média e $B$ é o tamanho do bucket (Figura 4). Desta forma é possível mostrar que o atraso máximo possui limite superior dado pela Equação 6.

Para controlar o atraso máximo aceitável na rede EON, foi assumido que cada lightpath é admitido através de um controle de admissão Leaky Bucket, que cada lightpath sempre tem maior capacidade que o tráfego agregado das camadas superiores (isto é, sem atrasos) e que o lightpath $l$ transmite a uma taxa de pico constante. Assim, $\sigma_{l}=\rho_{l}=$ $N_{l} T_{l}=R_{l}$.

A interpretação deste resultado sobre a visão do CALB é que a taxa de chegada do token é igual da taxa de chegada do fluxo. Desta forma, assumindo que o tráfego 
é admitido por um CALB, temos um limite superior para o atraso da transmissão do lightpath de acordo com a Equação 6. Para determinar se o lightpath l poderá violar seu atraso máximo de transmissão após perder slots, altera-se os parâmetros do CALB para computar o novo atraso através da Equação 6. Para isso, mantemos a taxa de transmissão do lightpath $R_{l}$ e ajustamos a taxa de chegada do token para ser igual a $N_{l} T_{l}$, onde $N_{l}<$ $N_{i}$ é a nova quantidade de slots do lightpath $l$. Esta ação é equivalente a comparar a taxa de transmissão original $\left(R_{l}\right)$ do lightpath $l$ a sua nova taxa de transmissão após a perda de slots. Este processo pode ser visualizado na Figura 4 . Por fim, comparamos $D_{n}$, o novo valor do atraso, com o valor pré-estabelecido $D_{M}$. Se $D_{n}>D_{M}$, a taxa de transmissão poderá exceder o deadline requisitado se o lightpath perder slots.

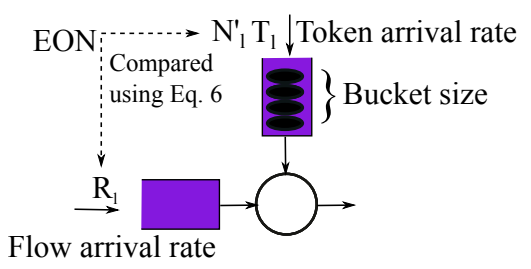

Figura 4. Controle de admissão Leaky Bucket.

\section{Exemplos numéricos}

Para avaliar o desempenho do algoritmo proposto foram realizadas simulações e comparações com as seguintes abordagens: Sem-Degradação (ND), Degradação-Total (FD), e Degradação-Parcial (PD). A ND não considera degradação de serviço, ou seja, considera-se o modo de operação padrão da rede. Já na FD, a requisição é degradada o máximo possível, isto é, deixando-a somente com dois slots: um para transmissão e outro como banda de guarda. As simulações foram realizadas em simulador próprio, utilizando o framework Simpy ${ }^{1}$.

$\mathrm{Na}$ abordagem $\mathrm{PD}$, as requisições degradadas perdem $30 \%, 50 \%$ ou $70 \%$ da sua largura de banda de forma aleatória. Por fim, a MDP degrada somente a quantidade de largura de banda permitida, ou seja, que não provocará violação do deadline estipulado. Cada abordagem possui sua versão com QoS proporcional, exceto a ND. Replicações independentes foram executadas para gerar um intervalo de confiança de 95\%. Para cada ponto nas curvas dos gráficos apresentados, foram executadas 10 simulações cada uma contendo 10000 requisições randomicamente escolhidas entre pares de nós origemdestino.

As requisições geradas seguiram um processo Poisson e foram distribuídas entre duas classes de serviços de maneira uniforme. As larguras de banda solicitadas foram de $5 G b p s$, 50Gbps e $150 G b p s$, e os valores para a quantidade de dados a transmitir foram $100 G b$ e $500 G b$. O tempo de duração de cada requisição foi definido como a razão entre a quantidade de dados a ser transferida e a largura de banda solicitada. O deadline para o término da transmissão de cada requisição foi escolhido de foma aleatória como $30 \%$, $50 \%$ ou $100 \%$ do seu tempo normal de transmissão. Assumiu-se fator de diferenciação entre classes de serviços $s_{1}=1$ e $s_{2}=2$, isto é, para cada lightpath degradado da classe 1 , duas requisições da classe 2 devem ser degradadas. Foram consideradas a topologia

\footnotetext{
${ }^{1}$ Simpy: Discrete event simulation for python, disponível em: https://simpy.readthedocs.io/en/latest/
} 
NSFNET, com 14 nós e 21 arestas (Figura 5), e a topologia USA, com 24 nós e 43 arestas (Figura 5). A capacidade adotada para cada enlace foi de 300 slots, cada um com 12,5GHz de frequência. Os formatos de modulação adotados foram BPSK, QPSK, 8-QAM e 16-QAM. Para a alocação de espectro foi utilizada a política First-Fit.

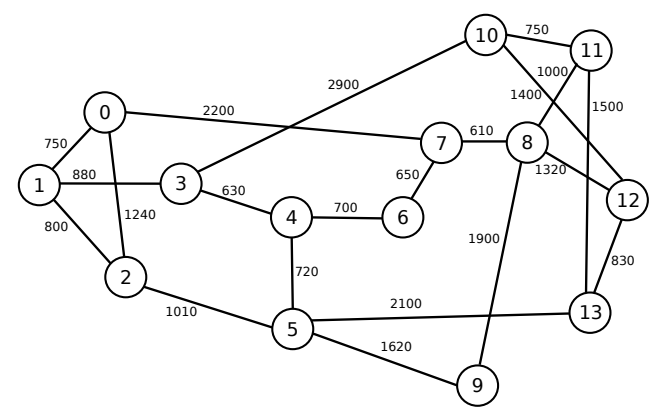

(a) NSFNET

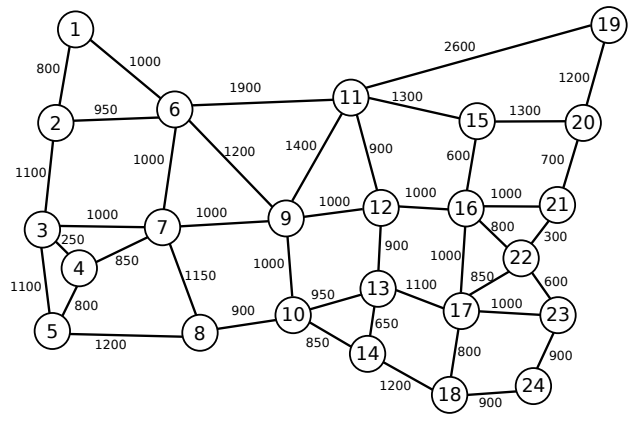

(b) USA

Figura 5. Topologias de rede utilizadas.

As métricas utilizadas para avaliação da proposta foram: probabilidade de bloqueio, violação de deadline, atraso médio e número médio de requisições degradadas para cada classe de serviço. A probabilidade de bloqueio é definida pela razão entre a quantidade de requisições bloqueadas e o total de requisições. A violação do deadline diz respeito às requisições estabelecidas que terminaram sua transmissão fora do tempo máximo estipulado. O atraso médio representa o incremento no tempo de transmissão provocado pela degradação.

Os experimentos foram conduzidos utilizando o modelo de planejamento fatorial completo de acordo com as definições propostas por [Jain 1991]. Os fatores e níveis adotados podem ser observados na Tabela 2 . Todos os resultados foram obtidos com intervalo de confiança, média e desvio padrão aferidos. A Figura 6 mostra os resultados obtidos, onde é possível perceber que o Fator A apresenta um maior impacto para a variável de resposta Violação do deadline $(41,9 \%)$, Número de requisições degradadas da classe 1 $(66,1 \%)$ e Bloqueio $(99,9 \%)$. Já o Fator B apresenta maior impacto para a variável de resposta Atraso médio (44\%). Por fim o Fator C apresenta maior impacto para a variável de resposta Número de requisições degradadas da classe 2 (39.1\%).

Tabela 2. Projeto Fatorial

\begin{tabular}{|l|c|c|}
\hline Fatores & Nível 1 & Nível 2 \\
\hline \hline $\mathrm{A}=$ Classe de Serviço & 1 & 2 \\
\hline $\mathrm{B}=$ Deadline de transmissão & $30 \%$ & $100 \%$ \\
\hline $\mathrm{C}=$ Carga na rede (erlangs) & 26 & 44 \\
\hline
\end{tabular}

A Figura 7 mostra a probabilidade de bloqueio para as topologias consideradas em função da carga na rede. Em ambas, os algoritmos que fazem uso da degradação apresentam menor probabilidade de bloqueio que o algoritmo ND, e esta diferença é ainda maior com o aumento da carga na rede. Verifica-se também, que os algoritmos que empregam QoS proporcional geram valores de probabilidade de bloqueio maiores se comparados 


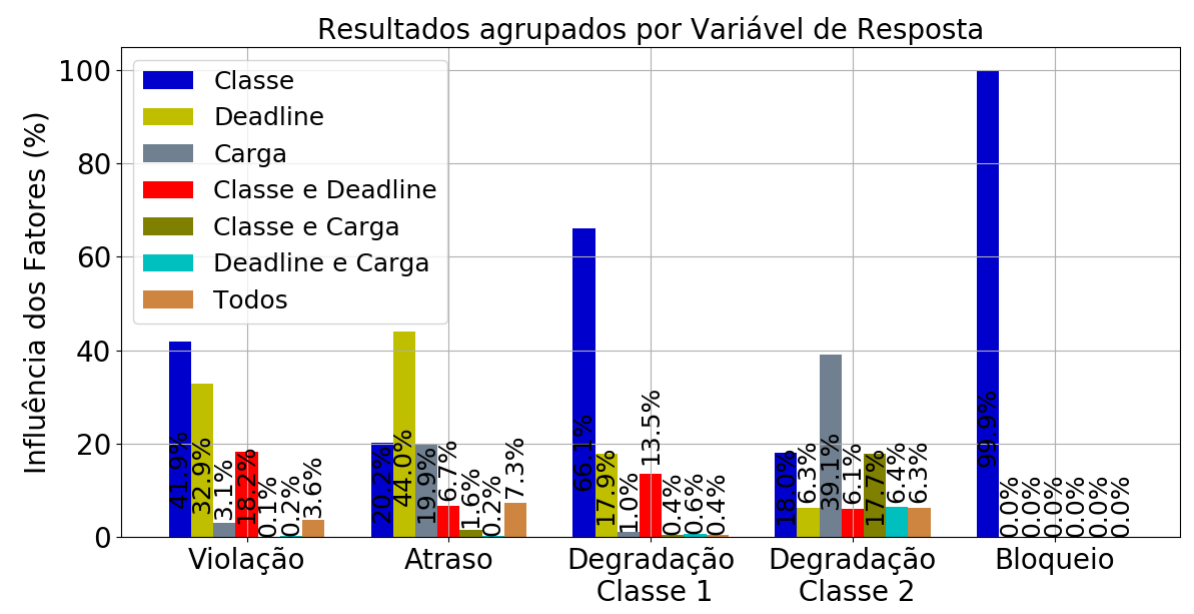

Figura 6. Projeto Fatorial.

aos algoritmos que não utilizam. Isto ocorre porque, com a utilização do QoS proporcional, uma requisição só é degradada se ela pertence à classe de serviço escolhida como descrito no Algoritmo 1, o que reduz as possibilidades de degradação se comparada às estratégias que não utilizam QoS proporcional. Outro fator a ser considerado é o fato das requisições degradadas não voltarem a sua largura de banda interior à degradação. Embora uma requisição degradada usando o algoritmo MDP esteja dentro de seus parâmetros de QoS estabelecidos, uma forma de solucionar esta situação seria criar uma heurística para retornar à largura de banda das requisições degradadas sempre que houvesse disponibilidade de recurso na rede. Como os resultados gerados para as topologias consideradas são semelhantes e devido à limitação de espaço, serão apresentados somente os resultados referentes à topologia NSFNET.

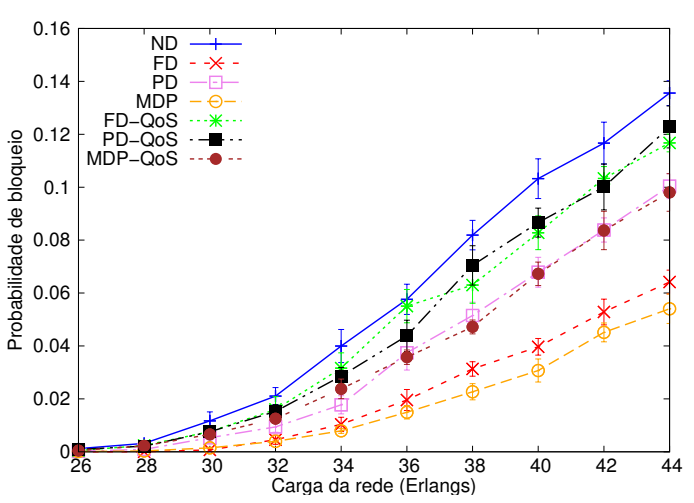

(a) Bloqueio NSFNET

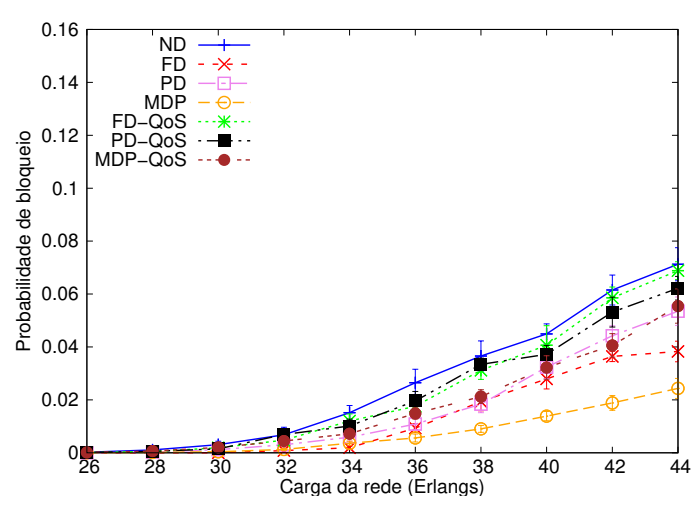

(b) Bloqueio USA

Figura 7. Probabilidade de bloqueio (\%) em função da carga na rede.

A Figura 8(a) apresenta o atraso médio causado pela degradação e a percentagem de conexões que violaram seu deadline em função da carga na rede. Como o algoritmo ND não degrada nenhuma das requisições atendidas, ele não provoca extensão no tempo de transmissão nem violação do deadline das requisições. Os algoritmos FD e FD-QoS, apresentam maiores porcentagem de violação e atraso médio, porque eles podem reduzir uma quantidade maior da largura de banda utilizada por uma requisição. Por outro lado, 
os algoritmos PD e PD-QoS apresentam melhores resultados se comparados com FD e FD-QoS, uma vez que a redução de largura de banda que eles podem realizar para uma determinada requisição é menor. Os algoritmos que apresentam melhores resultados são o MDP e o MDP-QoS. Isto ocorre porque estes algoritmos respeitam o deadline de cada requisição e só degradam a quantidade que não provocará a violação em excesso deste parâmetro. Assim, é evidenciada a importância deste algoritmo para a manutenção da qualidade de serviço.
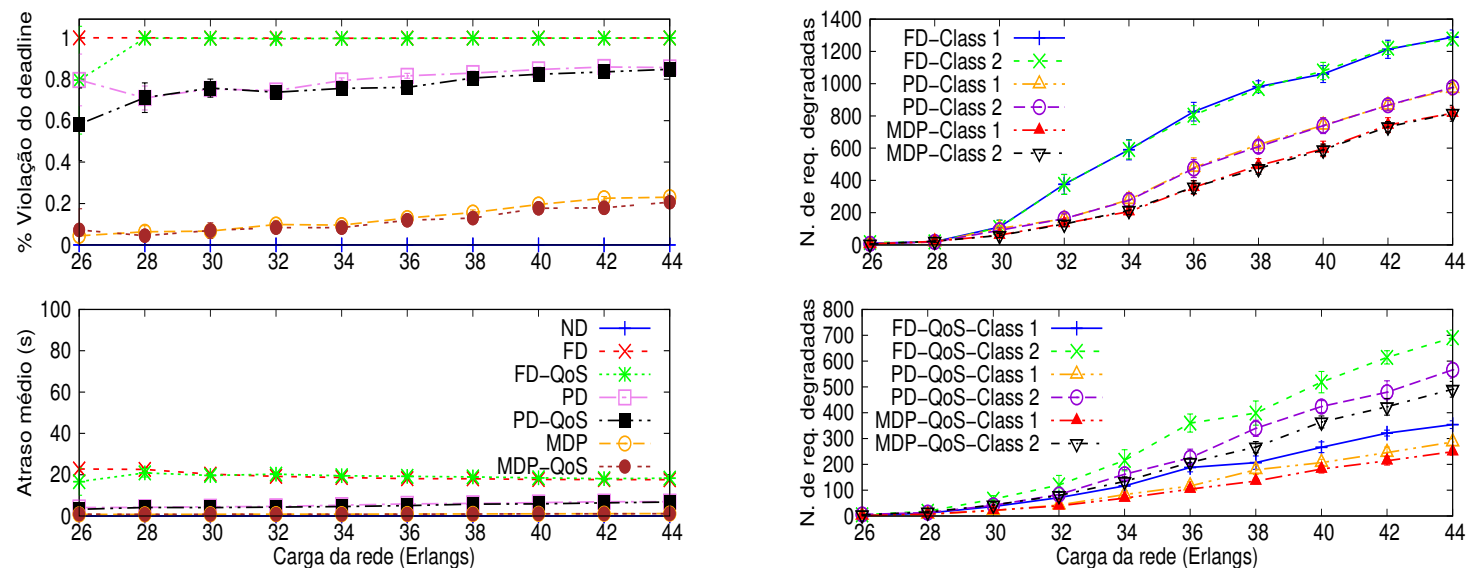

(a) Atraso médio e violação do deadline

(b) Número de requisições degradadas

Figura 8. Exemplos de degradação de serviço.

A Figura 8(b) apresenta o número de requisições degradadas para cada classe de serviço em função da carga na rede. Na parte superior da Figura 8(b) são apresentados os resultados dos algoritmos sem a utilização do QoS proporcional e na parte inferior o QoS é utilizado. É possível perceber que somente os algoritmos que utilizam o QoS proporcional são capazes de manter a proporcionalidade entre classes de serviços degradadas. Como os algoritmos FD, PD e MDP não seguem o modelo de QoS proposto, que nos experimentos foram adotados como duas degradações da classe de serviço 2 para cada uma da classe de serviço 1, temos as duas classes sendo penalizadas de foma igualitária. Além disto, a utilização do controle da violação do deadline e do atraso faz com que o algoritmo MDPQoS possua um número menor de requisições degradadas se comparado com o FD-QoS e o PD-QoS. É importante notar que os fatores de proporcionalidade podem ser alterados pelo operador da rede, dando maior flexibilidade no tratamento das classes de serviço.

\section{Conclusão}

Neste trabalho foi investigada a degradação de serviço com QoS Proporcional em Redes Ópticas Elásticas. Foi proposto uma estratégia online para a degradação de serviço modelada como um Generalized Processor Sharing com controle de admissão Leaky Bucket. Os resultados obtidos mostraram que a estratégia utilizada é capaz de reduzir a probabilidade de bloqueio além de garantir melhor tratamento entre diferentes classes de serviço.

\section{Referências}

Anoh, N. G., Babri, M., Kora, A. D., Faye, R. M., Aka, B., and Lishou, C. (2017). An efficient hybrid protection scheme with shared/dedicated backup paths on elastic optical networks. Digital Communications and Networks, 3(1):11 - 18. 
Chatterjee, B. C., Sarma, N., and Oki, E. (2015). Routing and spectrum allocation in elastic optical networks: A tutorial. IEEE Communications Surveys Tutorials, 17(3):17761800 .

Chen, Y., Qiao, C., Hamdi, M., and Tsang, D. H. K. (2003). Proportional differentiation: a scalable qos approach. IEEE Communications Magazine, 41(6):52-58.

Dovrolis, C. and Ramanathan, P. (1999). A case for relative differentiated services and the proportional differentiation model. IEEE network, 13(5):26-34.

Gerstel, O., Jinno, M., Lord, A., and Yoo, S. J. B. (2012). Elastic optical networking: a new dawn for the optical layer? IEEE Communications Magazine, 50(2):s12-s20.

Jain, R. (1991). The Art of Computer Systems Performance Analysis: Techniques for Experimental Design, Measurement, Simulation, and Modeling. Wiley.

Jinno, M., Takara, H., Kozicki, B., Tsukishima, Y., Sone, Y., and Matsuoka, S. (2009). Spectrum-efficient and scalable elastic optical path network: architecture, benefits, and enabling technologies. IEEE Communications Magazine, 47(11):66-73.

Napoli, A., Bohn, M., Rafique, D., Stavdas, A., Sambo, N., Poti, L., Nölle, M., Fischer, J. K., Riccardi, E., Pagano, A., Giglio, A. D., Moreolo, M. S., Fabrega, J. M., Hugues-Salas, E., Zervas, G., Simeonidou, D., Layec, P., D’Errico, A., Rahman, T., and Giménez, J. P. F. P. (2015). Next generation elastic optical networks: The vision of the european research project idealist. IEEE Communications Magazine, 53(2):152162.

Savas, S. S., Habib, M. F., Tornatore, M., Dikbiyik, F., and Mukherjee, B. (2014a). Network adaptability to disaster disruptions by exploiting degraded-service tolerance. IEEE Communications Magazine, 52(12):58-65.

Savas, S. S., Habib, M. F., Tornatore, M., and Mukherjee, B. (2014b). Exploiting degraded-service tolerance to improve performance of telecom networks. In Optical Fiber Communications Conference and Exhibition (OFC), 2014, pages 1-3.

Tomkos, I., Azodolmolky, S., Solé-Pareta, J., Careglio, D., and Palkopoulou, E. (2014). A tutorial on the flexible optical networking paradigm: State of the art, trends, and research challenges. Proceedings of the IEEE, 102(9):1317-1337.

Vadrevu, C. S. K., Wang, R., Tornatore, M., Martel, C. U., and Mukherjee, B. (2014). Degraded service provisioning in mixed-line-rate wdm backbone networks using multipath routing. IEEE/ACM Transactions on Networking, 22(3):840-849.

Yap, K.-K., McKeown, N., and Katti, S. (2012). Multi-server generalized processor sharing. In Int. Teletraffic Congress (ITC), pages 29:1-29:8.

Zhang, G., Leenheer, M. D., Morea, A., and Mukherjee, B. (2013). A survey on ofdm-based elastic core optical networking. IEEE Communications Surveys Tutorials, 15(1):65-87.

Zhong, Z., Li, J., Hua, N., Figueiredo, G. B., Li, Y., Zheng, X., and Mukherjee, B. (2016). On QoS-assured degraded provisioning in service-differentiated multi-layer elastic optical networks. In IEEE Global Communications Conference (GLOBECOM), pages 1-5. IEEE. 